\title{
USO DE FLAVONOIDES (HESPERIDINA) EN EL TRATAMIENTO DE VENAS VARICOSAS VESICALES SANGRANTES
}

\author{
E.A. GRANADOS LOARCA \\ Clínica de Urología. Torre Profesional. Quetzaltenango. Guatemala. \\ Actas Urol Esp. 27 (9): 732-734, 2003-07-24
}

\section{RESUMEN}

USO DE FLAVONOIDES (HESPERIDINA) EN EL TRATAMIENTO DE VENAS VARICOSAS VESICALES SANGRANTES

OBJETIVO: Informar del uso de los Flavonoides (Hesperidina) en el tratamiento de varices vesicales, y su eficacia.

PACIENTES: Se informa de 4 casos que acudieron por hematuria, y que en la cistoscopia se observó varices vesicales, sangrantes en 2 casos; en ellos se utilizó Hesperidina $150 \mathrm{Mg}$. bid o tid por 30 días.

RESULTADOS: Fueron buenos, en los 2 casos que sangraban desapareció la hematuria y en los 4 casos en la cistoscopia de control las varices habian desaparecido.

CONCLUSIONES: El tratamiento antivaricoso o anti-hemorroidal puede ser útil en el tratamiento de varices vesicales. Se debe de dar como mínimo por 30 días

PALABRAS CLAVE: Varices vesicales. Hesperidina. Antivaricoso.

\section{ABSTRACT}

TREATMENT OF BLADDER VARICOSE VEINS WITH FLAVONOIDS (HESPERIDINA)

OBJECTIVE: To inform of the use of the Flavonoids in the treatment of bladder varicose veins, and their effectiveness.

PATIENT: It is informed of 4 cases that went for haematuria, and that in the cistoscopy one observes bladder varicose veins, bleeding in 2 cases, in them you uses Hesperidina $150 \mathrm{mg}$ bid or tid for 30 days.

RESULTS: They were good, the 2 cases that bled the haematuria disappeared and in the 4 cases in the control cistoscopy the varicose veins had disappeared.

CONCLUSIONS: The treatment antivaricose or antihemorrhoidal can be useful in the treatment of bladder varicose veins. It should be given as minimum by 30 days.

KEY WORDS: Bladder varicose veins. Hesperidina. Antivaricose.

$L^{\circ}$ os flavonoides se han utilizado en el trataAmiento de insuficiencia venosa crónica, dermatitis varicosa, úlceras varicosas, linfedema, hemorroides, varicoflebitis y trastornos retinianos vasculares. En este reporte se incluyen 4 casos con varices vesicales en quienes se utilizó flavonoides (Hesperidina).

\section{CASOS CLÍNICOS}

Caso $n^{o}$ 1: Paciente de 38 años femenina, que acudió por presentar hematuria de 5 días. Antecedentes de hematuria en 2 ocasiones que cedieron sin tratamiento. Al examen físico se observó venas varicosas en las extremidades inferiores, las cuales se había infiltrado en 2 ocasiones, 
y venas varicosas en el periné. Uro-análisis microhematuria (8-10 eritrocitos por campo). Ultrasonido renal y vesical, y la urografía intravenosa fueron normales. Mediante la cistoscopia se observó varices vesicales; algunas sangraban suavemente.

Se le dio tratamiento médico con Hesperidina $150 \mathrm{Mg}$. (Ciclo 3 Forte ${ }^{\circledR}$ ) tid por 15 días y luego bid por otros 15 días con lo cual desapareció la hematuria; en la cistoscopia de control a las 4 semanas de finalizar el tratamiento la vejiga fue normal.

Caso $n^{\circ}$ 2: Paciente de 40 años femenina, post-parto que acudió por presentar hematuria franca de 2 días. Antecedentes de hemorroides sin tratamiento. Al examen físico se observó venas varicosas en las extremidades inferiores, periné, y hemorroides. Uro-análisis, microhematuria campos llenos de eritrocitos. Ultrasonido renal y vesical, y la urografía intravenosa demostraron coágulos intravesicales. Mediante la cistoscopia se observó varices vesicales sangrantes, se cauterizó algunas, pero no se pudo cauterizar todas ya que suelen sangrar más, por lo que se le dio tratamiento médico con Hesperidina $150 \mathrm{Mg}$. (Ciclo 3 Forte ${ }^{\circledR}$ ) tid por 15 días y luego bid por otros 15 días con lo cual desapareció la hematuria; en la cistoscopia de control a las 6 semanas de finalizar el tratamiento la vejiga fue normal.

Caso no 3: Paciente de 40 años femenina, que acudió por presentar hematuria de 15 días. Antecedentes de hematuria en 4 ocasiones que cedieron al tomar tratamiento para las hemorroides, el cual tomó por 2 semanas. La hematuria volvió tres meses después de no tomar el tratamiento para las hemorroides. La paciente tenía hemorroides y venas varicosas vulvares. Uroanálisis microhematuria de 10-15 eritrocitos por campo. Ultrasonido renal y vesical, y la urografía intravenosa fueron normales. Mediante la cistoscopia se observó varices vesicales las cuales no sangraban. Se le dio tratamiento médico con Hesperidina $150 \mathrm{Mg}$ (Ciclo 3 Forte ${ }^{\circledR}$ ) bid por 30 días con lo cual desapareció la hematuria; en la cistoscopia de control a las 4 semanas de finalizar el tratamiento la vejiga fue normal.
Caso no 4: Paciente de 43 años femenina, post-parto que acudió por presentar hematuria franca de 2 días. Antecedentes de hemorroides sin tratamiento. Al examen físico se observó venas varicosas en las extremidades inferiores, vulva y periné. Uro-análisis microhematuria de 5 eritrocitos por campo. Ultrasonido renal y vesical, y la urografía intravenosa fueron normales. Mediante la cistoscopia se observó varices vesicales las cuales no sangraban. Se le dio tratamiento médico con Hesperidina $150 \mathrm{Mg}$ (Ciclo 3 Forte () bid por 30 días, la hematuria no volvió a aparecer; en la cistoscopia de control a las 8 semanas de finalizar el tratamiento la vejiga fue normal.

\section{DISCUSIÓN}

La presencia de varices en la vejiga se deben a los mismos factores que condicionan que aparezcan varices en las extremidades inferiores, pélvicas, hemorroides etc. Cuando existen varices, la inflamación se caracteriza por una permeabilidad vascular aumentada asociado a vasodilatación, seguida de una migración celular como resultado de la quimiotaxis de las células hacia el sitio de la inflamación.

Todos los mediadores biológicos que modifican la vasoactividad, aumentan la permeabilidad microvascular o inician la migración y degranulación leucocitaria en el espacio intercelular, desempeñando un papel en la formación del edema. Una vez iniciada la vasodilatación entran en acción la bradiquinina, la histamina, y la PGE2, siendo estos los principales agentes responsables del aumento de la permeabilidad microvascular. Las linfoquinas (IL1, IL8, y TNF) y el LTB4 ejercen los efectos quimiotácticos más intensos. Finalmente, los radicales libres derivados del oxígeno producidos en el medio extracelular principalmente por los fagocitos (células polimorfonucleares y macrófagos) contribuyen de varias maneras a las lesiones inflamatorias; lo cual induce histolisis (fibroblastos, células endoteliales, eritrocitos), que aumentan la permeabilidad de las paredes vasculares y perpetúan el proceso inflamatorio ${ }^{1,2}$.

Las varices vesicales pueden ser causa de hematuria no explicable en especial en aquellos pacientes con problemas venosos tales como 
varices de miembros inferiores, hemorroides etc, los cuales pueden aumentar en el transcurso o posterior al parto.

Los Flavonoides tienen su acción farmacológica sobre los procesos inflamatorios. Entre ellos están: la Diosmina que es una Flavona; la Herperidina que es una Flavonona, componentes específicos en el tratamiento de los trastornos perivenosos, proinflamatorios. Los flavonoides son conocidos como inhibidores de la actividad de las enzimas involucradas en la transformación de los ácidos grasos poliinsaturados de la membrana citoplasmática. Útiles en la estasis venosa. La Hesperidina tiene propiedades venotónicas, vasculoprotectoras, flevolíticas, y antivaricoso. $\mathrm{Su}$ acción es sobre la microcirculación, aumentan la resistencia y la permeabilidad, y protegen el endotelio vascular. Además de estas propiedades antiinflamatorias, los flavonoides tienen también efectos analgésicos y antiagregantes plaquetarios. La diosmina y herperidina, reducen el edema inducido por obstrucción venosa ${ }^{3,4}$.

La aparente paradoja de la combinación de estas propiedades en la misma molécula se podría explicar por la interferencia con la producción de radicales libres oxigenados.

El tratamiento cuando existen hemorroides o procesos tromboflebíticos se suele dar durante 30 días con dosis de $100 \mathrm{mg} / \mathrm{Kg} /$ día (vía oral), lo cual reduce la extravasación de plasma, y reduce la invasión del granuloma por los leucocitos.

Exámenes histológicos han demostrado un retraso de la diapédesis de los leucocitos, histiocitos, y macrófagos hacia el sitio de la inflamación. Se ha observado una disminución del edema perivascular después de utilizar flavonoides los cuales reducen la síntesis de PGE2 y del Tromboxano TxA2. El uso de los flavonoides bid o tid, reducen la permeabilidad vascular inducida por la bradiquina o la histamina ${ }^{5}$.

Los flavonoides se han utilizado en el tratamiento de insuficiencia venosa crónica, dermatitis varicosa, úlceras varicosas, linfedema, hemorroides, varicoflebitis, trastornos retinianos vasculares y varices vesicales.
Cuando ocurre hematuria los exámenes de ultrasonido y urografía intravenosa puede que no demuestren más que la posibilidad de coágulos intravesicales, siendo necesario efectuar la cistoscopia, donde se puede observar la causa de la hematuria. En los casos de varices sangrantes se puede utilizar la coagulación con bola como se efectuó en un caso pero sin mayor resultado por el tipo de venas sangrantes, ya que son gruesas y largas. El uso del Láser podría ser otra posibilidad, pero en la clínica no contamos con ella.

Entre las contraindicaciones del uso de Flavonoides están: hipersensibilidad, reacciones alergias, cefalea, y rubores; que desaparecen al suspender el tratamiento.

\section{CONCLUSIONES}

El uso de Flavonoides en el tratamiento de varices vesicales, sangrantes o no, puede ser una alternativa terapéutica.

\section{REFERENCIAS}

1. LEE T, MATTELIANO ML, MIDDLETON E.: Effect of quercetin on human PMNL lysomal enzyme release and phospholipid metabolism. Life Sci 1982; 31: 2.765 .

2. BAUMAN J, BRUCHHAUSEN F, WURM G.: Flavonoids and related compounds as inhibitors of arachidonic acid peroxidation. Prostaglandin 1980; 20: 627.

3. MOORE PK, GRIFFITHS RJ, LOFTS FJ.: The effect of some flavone drugs on the conversion of prstacyclinto 6-oxoprostaglandin E1. Biochem Pharmacol 1983; 32: 2.813 .

4. LANDOLI R, MOWER R, STEINER M.: Modification of platelet function and arachidonic acid metabolism by flavonoids. Biochem Pharmacol 1984; 33: 1.525.

5. VILLAR A, GASCO MA, ALCARAZ MJ.: Anti-inflammatory and antiulcer properties of hypolaetin-8-glucoside, a novel plant flavonoid. $J$ Pharm Pharmacol 1984; 36: 820 .

Dr. E.A. Granados Loarca

31 Avda. "F" 14-60 Zona 7

Cond. Villas de San Martín (Guatemala)

(Trabajo recibido el 7 mayo de 2003) 